\title{
Cardiac rhabdomyomata in tuberous sclerosis: their course and diagnostic value
}

\author{
H C SMITH, ${ }^{*}$ G H WATSON, $\dagger$ R G PATEL,$\dagger$ AND M SUPER* \\ †Departments of Cardiology and *Clinical Genetics, Royal Manchester Children's Hospital
}

SUMMARY Echocardiography was performed in 60 people with tuberous sclerosis to ascertain the prevalence and course of cardiac rhabdomyomata at different ages. Twenty five (58\%) of 43 children had tumours, but only three $(18 \%)$ of 17 adults. The tumours tended to remain the same size through childhood. Only three infants were included but evidence from this and some published case reports suggest that the tumours tend to regress in early infancy and again in adolescence. The prevalence of tumours in young infants with tuberous sclerosis is likely to be considerably above $50 \%$. As other signs of tuberous sclerosis are usually absent at this age echocardiography may afford the most useful diagnostic test in early infancy.

It has been known for many years that people with tuberous sclerosis may have cardiac rhabdomyomata as part of the syndrome, but assessment of the prevalence of the tumours has depended in the past largely on necropsy studies. These studies have suggested a high mortality from rhabdomyomata in the first few years of life with $78 \%$ of deaths in the first year. It has been said that $37-50 \%$ of these deaths are associated with tuberous sclerosis but this is probably an underestimate as other diagnostic features of tuberous sclerosis may not appear until later childhood. ${ }^{12}$ Conversely, necropsy studies in people known to have tuberous sclerosis have suggested up to $30 \%$ with rhabdomyomata ${ }^{1}$ but these figures may also be biased.

Two dimensional echocardiography offers a simple non-invasive method that shows cardiac tumours very well, and two small studies have found cardiac tumours in eight of 16 and seven of 11 people with tuberous sclerosis respectively. ${ }^{3}{ }^{4}$ The present study was carried out in the hope of assessing the prevalence of these tumours at different ages and also because of the impression that some tumours may regress.

\section{Methods}

Patients with tuberous sclerosis and their families were contacted through the Tuberous Sclerosis Association of Great Britain and the departments of clinical genetics and paediatric neurology in the North West region. Patients were examined to confirm the diagnosis, and echocardiography carried out by one or both paediatric cardiologists using an ATL (Advanced Technology Laboratories) apparatus, with appropriate recordings. Where possible first degree relatives also had echocardiograms and were examined for cutaneous evidence of tuberous sclerosis; this included a search for hypomelanotic macules with a Wood's light.

We felt it important to explain our findings in detail to parents because of the natural anxiety otherwise engendered.

Three of the invited families did not attend, and four of those attending did not have signs that satisfied strict diagnostic criteria for tuberous sclerosis ${ }^{5}$; they were therefore excluded as were three people with tuberous sclerosis whom we could not persuade to undergo echocardiography. Altogether 60 people with unequivocal tuberous sclerosis had satisfactory scans. There were 29 males and 31 females aged up to 41 years. None had any significant murmurs (except one infant described later) or had ever had any cardiac symptoms.

\section{Results}

A total of $28(47 \%)$ people (14 males, 14 females) had cardiac tumours shown by echocardiography, while 32 ( 15 males, 17 females) did not. As others have found, ${ }^{1-4}$ most tumours were related to the ventricles; in 17 they were multiple. There was no correlation between the presence of cardiac tumours and the extent of the skin manifestations including hypomelanotic spots, degree of mental handicap, or convulsions. 
Thirty seven first degree relatives ( 33 adults and four children) were examined. None had any skin manifestations or symptoms of tuberous sclerosis. No cardiac tumours were found in the adults but one infant had two $8 \mathrm{~mm}$ tumours in the left ventricle.

Table 1 shows the prevalence of tumours at different ages. There was no significant difference in the prevalence of tumours through childhood, but children under 16 years were significantly more likely to have tumours than adults $(25$ of 43 children but only three of 17 adults; $\left.\chi^{2}=8 \cdot 02, p<0 \cdot 01\right)$. Table 2 shows that the mean number of tumours per person in those who have them did not seem to vary significantly at different ages.

The mean diameter of tumours in those who had any was assessed by taking the mean of two largest dimensions for each tumour and averaging these for the age groups. Table 2 shows no significant difference between the absolute sizes of the tumours at different ages, although the trend is for diminution with increasing age. As the linear dimensions of the cardiac chambers of an adult are almost twice those of a child of 2 years there is clearly a relative diminution in tumour size.

In most cases the tumours were easily seen (fig 1), though in a few they were found only after a careful search. Many tumours were highly reflectile, but a few undoubted tumours did not seem very different from adjacent myocardium; we feel that the difference may be related to maturation (see below). Some quite reflectile tumours, however, were seen in older children and adults. Some reflectile areas are commonly seen in normal myocardium and these need to be distinguished from small intramural

Table 1 Prevalence of intracardiac tumours in subjects with tuberous sclerosis

\begin{tabular}{lccccc}
\hline & \multicolumn{5}{l}{ Age (years) } \\
\cline { 2 - 6 } & $<6$ & $6-<12$ & $12-<18$ & $>18$ & Total \\
\hline Tumours seen & 9 & 8 & 8 & 3 & 28 \\
Tumours not seen & 5 & 9 & 4 & 14 & 32 \\
\hline Total & 14 & 17 & 12 & 17 & 60 \\
\hline
\end{tabular}

tumours; the normal areas are not rounded and may look linear in some views. The left ventricular papillary muscles were a source of difficulty at times; in normal people they may be denser than the mural myocardium and occasionally they may be

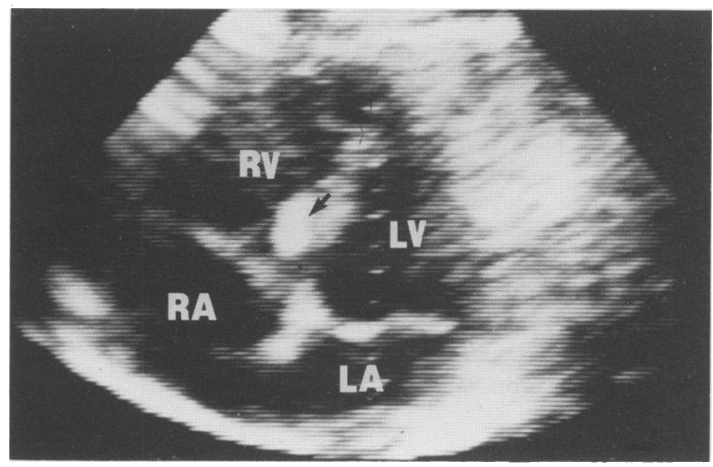

Fig 1 A child aged 10 years. Four chamber view of the heart showing a tumour attached to the right ventricular side of the ventricular septum. (In the figures the long arrows show the left ventricular wall and the short arrows show tumours; scale marks are $1 / 2$ and $1 \mathrm{~cm}$. AOR, aorta; $L A$, left atrium; $L V$, left ventricle; $R A$, right atrium; and $R V$, right ventricle.)

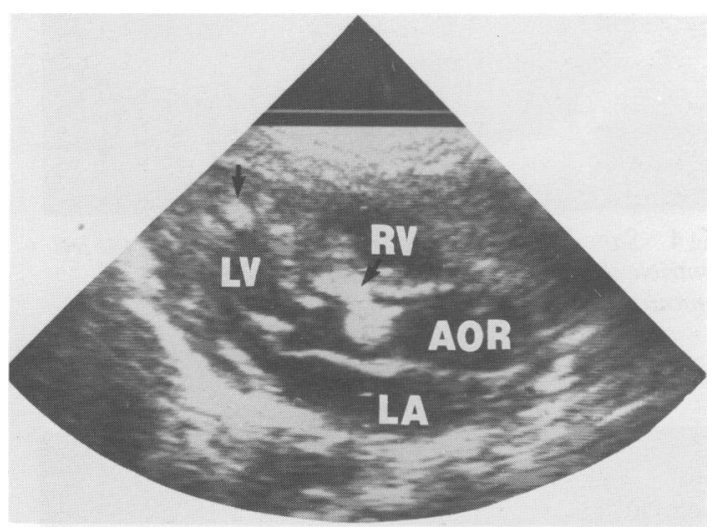

Fig 2 Boy aged 1 week. Long axis view of the left ventricle showing multiple tumours in the ventricular septum, one projecting into the left ventricular outflow.

Table 2 Mean number and diameter of tumours at different ages

\begin{tabular}{lllll}
\hline & \multicolumn{1}{l}{ Age (years) } & & & \\
\cline { 2 - 5 } & $<6$ & $6-<12$ & $12-<18$ & $>18$ \\
\hline No with tumours: & 9 & 8 & 8 & 3 \\
Mean (SD) tumours/person & $2 \cdot 7(1.58)$ & $2 \cdot 6(0.92)$ & $2 \cdot 5(0.53)$ & $3.0(1.0)$ \\
Mean (SD) diameter (mm) & $7 \cdot 89(1.78)$ & $6.55(2.31)$ & $7 \cdot 58(3.56)$ & $5 \cdot 72(3.27)$ \\
\hline
\end{tabular}




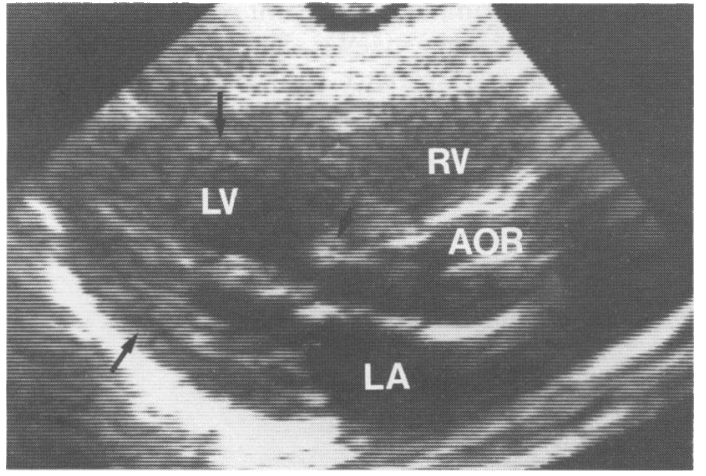

Fig 3 Same child aged 6 months. Long axis view of the left ventricle. The previously obstructing tumour (fig 2) is less dense and smaller; it is mobile and is shown in its diastolic position.

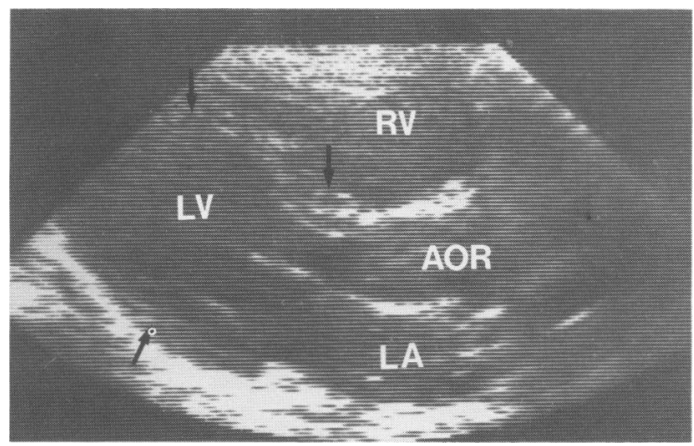

Fig 4 Same child aged 4 years. Long axis view of the left ventricle showing a normal left ventricle and no tumours.

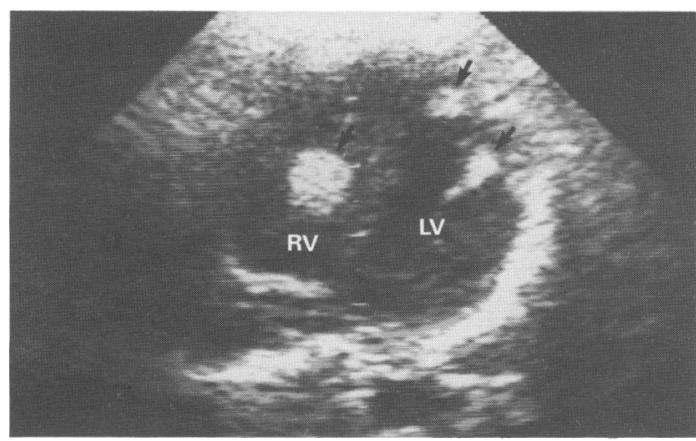

Fig 5 Same child aged 1 week. Oblique view of the ventricles showing tumours in the left ventricular wall and right ventricular cavity.

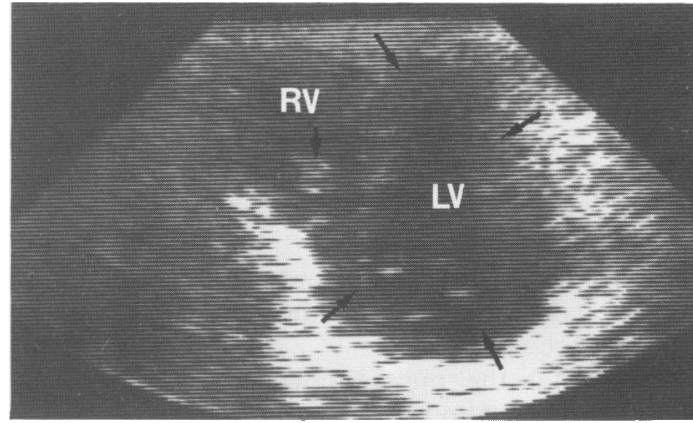

Fig 6 Same child aged 1 year. Oblique view of the ventricles. The right ventricular tumour is smaller and indistinct; no tumours can be seen in the left ventricular wall.

strikingly wide or multiple. We included only undoubted tumours but wondered if three other odd shaped papillary muscles might represent maturing tumours. Authors of some necropsy studies have commented on the tendency of tumours to be more obvious in the left ventricle and papillary muscles. ${ }^{6}$

Three infants with cardiac tumours had sequential studies, and in each there was regression of the tumours, some of which disappeared. Figs 2-6 show the changes in one of these children over a period of four years. Multiple tumours have regressed, leaving only a single $8 \mathrm{~mm}$ tumour in the right ventricular cavity. The tumours seen in figs 3 and 6 are clearly much less dense than the same tumours seen earlier in figs 2 and 5 respectively.

\section{Discussion}

The overall prevalence of cardiac tumours in these people with tuberous sclerosis is similar to that reported in the two other published ultrasound studies. ${ }^{34}$ The low prevalence in adults with tuberous sclerosis has not previously received comment, though the eight cases of Bass et al show a similar trend. ${ }^{3}$ The difference seems unlikely to be entirely the result of poorer visualisation in adults, for in all the other age groups easily visible $10 \mathrm{~mm}$ tumours were present in at least half of those who had tumours. As rhabdomyomata are a very rare cause of death at this age then presumably there has been regression of the tumours. Although the word rhabdomyomata suggests new growth, histologists have for many years regarded them as hamartomata (Greek 'hamartia', error of judgment) that do not progress or become malignant. Steinbiss described lesions in older patients, which could well have been remnants of previously larger tumours. ${ }^{7}$ 
We have found no information on the course of tumours in adolescence, though there is a case report of an asymptomatic childhood rhabdomyoma whose size remained unchanged over a period of three years. ${ }^{8}$ There are case reports, however, of 12 infants later shown to have tuberous sclerosis-all in fact under 2 months old when first seen-who had a later review ${ }^{8-15}$; in 11 of these the tumours regressed or even disappeared, while in one the tumours were the same size 18 months later. ${ }^{15}$ Unfortunately our series included only three infants but regression occurred in all of them. It seems likely, therefore, that regression is the usual course not only in early adult life but also in infancy, and that the prevalence of tumours in neonates is higher than the $50 \%$ seen in older children.

This has important implications for management of symptomatic tumours. Obstructive symptoms or arrhythmias due to a rhabdomyoma in tuberous sclerosis are rare, and did not occur in our series, but some authors have proposed urgent surgery for symptomatic cardiac tumours despite the high mortality. ${ }^{8}$ The chances of tumour regression or relative lack of growth, however, suggest a conservative approach. ${ }^{10} 12$ The boy in this series referred to in figs 2-6 developed a murmur suggesting subaortic stenosis, but he remained quite well and the murmur disappeared as the tumour regressed.

As far as the question of whether an infant or fetus with a cardiac tumour will turn out to have tuberous sclerosis is concerned, the quoted risk of $50 \%{ }^{2}$ is probably an underestimate as it derives from postmortem studies particularly in neonates in whom the diagnosis of tuberous sclerosis may be difficult to establish. It seems very probable that nearly all multiple tumours are rhabdomyomata and nearly all these are due to tuberous sclerosis. ${ }^{16} \mathrm{~A}$ total of $25 \%$ were single in our series but as rhabdomyomata are by far the commonest tumour in infancy then even a single tumour found incidentally by echocardiography is in the absence of other clues also likely to be a rhabdomyoma and probably due to tuberous sclerosis.

The lack of changes in the skin or on computed tomography in an infant with cardiac tumours should not lead to false reassurance. Although cardiac tumours have not been described as a sole manifestation of tuberous sclerosis in adults, ${ }^{17}$ three infants in this study had no other signs of tuberous sclerosis at presentation, though they appeared later in two of the three. We have found published reports of six neonates with cardiac tumours and later signs of tuberous sclerosis in whom pale spots were mentioned; the spots appeared before the age of 5 months in only one baby. ${ }^{10} 11151819$ In the screening of adult relatives the value of cardiac ultrasound is clearly less than in infancy: in the present series tumours were not found in the apparently unaffected relatives and were relatively infrequent in even affected adults. In childhood and particularly in infants, however, cardiac ultrasound may well be helpful because of the higher prevalence of tumours; it would, for example, be particularly helpful in babies who present with infantile spasms, but who have not yet developed any aetiological evidence, so as to allow early diagnosis and appropriate family genetic advice for some of the $25 \%$ who eventually show features of tuberous sclerosis. ${ }^{20}$ Echocardiography, when indicated, should not be delayed, lest the tumours regress. Fetal echocardiography also seems likely to afford a reasonable yield where there is a relevant family history, particularly if the prevalence in infants should prove to be high. 1011151819

We are most grateful to the parents and children who took part in the study, to Dr EM Carr-Saunders for data on one family, and to many other paediatricians and family doctors. In particular we thank Mrs Ann Hunt and the Tuberous Sclerosis Association for much help, encouragement, and financial support.

\section{References}

1 Fenoglio JJ Jr, McAllister HA Jr, Ferrans VJ. Cardiac rhabdomyoma: a clinico-pathological and electron microscopic study. Am J Cardiol 1976;38:241-51.

2 Batchelor TM, Maun ME. Congenital glycogenic tumours of the heart. Archives of Pathology 1945;39:67-73.

3 Bass JL, Breningstal G, Swaiman KF. Echocardiographic incidence of cardiac rhabdomyoma in tuberous sclerosis. $\mathrm{Am} \mathrm{J}$ Cardiol 1985;55:1379-82.

4 Gibbs JL. The heart and tuberous sclerosis: an echocardiographic and electrocardiographic study. Br Heart J 1985;54:596-9.

5 Gomez MR. Tuberous sclerosis. New York: Raven Press, 1979.

6 Thibault JH, Manuelidis EE. Tuberose sclerosis in a premature infant. Neurology 1970;20:139-46.

7 Steinbiss W. Zur Kenntnis der Rhabdomyome des Herzens und Ihrer Beziehungen zur Tuberosen Gehirnsklerose. Virchows Archiv 1923;243:22-38.

8 Khattar H, Guerin R, Fouron JC, Stanley P, Kratz C, Davignon A. Heart tumours in children. Report of three cases with favourable spontaneous courses. Arch Mal Coeur 1975;68: $419-29$.

9 Foster ED, Spooner EW, Farina MA, Shaher RM, Alley RD. Cardiac rhabdomyoma in the neonate: surgical management. Ann Thorac Surg 1984;37:249-53.

10 Gillor A, Stock G, Schuster D, et al. Diagnostik und Behandlung der Kardialen Rhabdomyome. Monatsschr Kinderheilkd 1986;134:445-9.

11 Gresser CD, Shime J, Rakowski H, Smallhorn JF, Hui A, Berg JJ. Fetal cardiac tumor: a prenatal echocardiographic marker for tuberous sclerosis. Am J Obstet Gynecol 1987;156:689-90.

12 Stijns M, Lintermans J, Tremouroux M, Vliers A. Spontaneous disappearance of aortic subvalvar obstruction in young infants with tuberous sclerosis. Pediatr Cardiol 1982;3:88-9.

13 Casta A. Tuberous sclerosis and Wolff-Parkinson-White syndrome. J Pediatr 1986;109:399.

14 Marx GR, Bierman FZ, Matthews E, Williams R. Twodimensional echocardiographic diagnosis of intra-cardiac masses in infancy. J Am Coll Cardiol 1984;3:827-32. 
15 Journel H, Roussey M, Plais MH, Milon J, Almange C, Le Marec B. Prenatal diagnosis of familial tuberous sclerosis following detection of cardiac rhabdomyoma by ultrasound. Prenat Diagn 1986;6:283-9.

16 Pomerance A, Davies MJ. The pathology of the heart. Oxford: Blackwell, 1975.

${ }^{17}$ Fryer AE, Chalmers A, Connor JM, et al. Evidence that the gene for tuberous sclerosis is on chromosome 9. Lance 1987;i:659-60.

${ }^{18}$ Muller L, De Jong G, Falck V, et al. Antenatal ultrasonographic findings in tuberous sclerosis. $S$ Afr Med J 1986;69:633-8.
19 Wilson RD, Hall JG, McGillivray BC. Tuberous sclerosis: case report and investigation of family members. Canad Med Assoc J 1985;132:807-9.

${ }^{20}$ Pampiglione G, Pugh E. Infantile spasms and subsequent appearance of tuberous sclerosis syndrome. Lancet 1975;ii:1046.

Correspondence to Dr GH Watson, Royal Manchester Children's Hospital, Pendlebury, Manchester M27 1HA.

Accepted 27 June 1988 